\title{
Use of complementary and alternative medicine by children in italy - a status quo
}

\begin{abstract}
Introduction: Although the popularity of complementary and alternative medicine (CAM) in Italy has risen in the last decade, information about its use by paediatric patients is still scarce.

Methods: A narrative, non-systematic review was performed by combining expert opinions with a thorough and balanced review of available evidence. A literature search of local as well as international published surveys was performed to present an overview about use of CAM in children in Italy. Furthermore, integration of CAM within different levels of healthcare in paediatrics was described.
\end{abstract}

Results: Twenty surveys were found between 1995 and 2011. Most surveys in the general population showed a CAM use between $30-48 \%$ and a similar prevalence in children. Homeopathy proved to be the most frequently used CAM followed by phytotherapy, acupuncture, Anthroposophic medicine, manual treatment (chiropractic and osteopathy) and oriental medicine. Although there are many outpatient clinics and inpatient wards offering CAM and many educational programs for medical professionals, there is still no national regulation on the handling of CAM.

Conclusion: CAM use in Italy seems to be as high as in other European countries in adults and in children. But in contrast to most other countries, several surveys have been conducted to describe CAM use and its users and multiple efforts have been made to clarify its role in the health system. Local CAM experts are in the process of creating a national law which corresponds with future guidelines of the European Union.

Keywords: complementary and alternative medicine, anthroposophic, homeopathy, herbal,acupuncture, mind-body, children, paediatrics, outpatient, inpatient, primary care
Volume 4 Issue I - 2016

\author{
Domenico Careddu,' Francesco Macr,' \\ Matteo Moretti, ${ }^{2}$ Rebekka Bailey, ${ }^{3}$ Alfred \\ Langler, ${ }^{3,4}$ Tycho Jan Zuzak, ${ }^{4,5}$ \\ 'Vice President of the Italian Society of Natural Medicine, Italy \\ 2Department of Pediatrics, Sapienza University of Rome, Italy \\ 3Faculty of Health, University of Witten/Herdecke, Germany \\ ${ }^{4}$ Department for Integrative Paediatric and Adolescent Medicine, \\ Gemeinschaftskrankenhaus, Germany \\ ${ }^{5}$ Department of Paediatric Oncology and Haematology, \\ University Children
}

Correspondence: Tycho Zuzak, Department of Paediatric Oncology and Haematology, Complementary and Alternative Medicine Research, University Children, Tel +49/ (0) 201723 84496,Email tycho.zuzak@uk-essen.de

Received: May 18, 2016 | Published: June 16, 2016
Abbreviations: CAM, complementary and alternative medicine

\section{Introduction}

Complementary and alternative medicine (CAM) is often used by patients in Europe. ${ }^{1}$ Diverse cultural; ethnic and historical preconditions in the various European countries result in broad differences between the types of CAM practiced or the extent of CAM use in the different European populations. ${ }^{2}$ Currently; CAM is provided primarily by general practitioners and non-medical therapists. ${ }^{3}$ Some efforts have been made in the last decade to explore the side-effects and potential benefits of CAM use; and improve the quality by augmenting education in CAM; however; these primarily targeted the use by adults. ${ }^{4-7}$ The increasing integration of CAM into hospitals and medical facilities has created a demand for unification and quality assurance on a European level. ${ }^{8}$ Despite this increasing harmonisation and unification of the European legislation within the European Union framework; there are still different conditions in the health policies in every EU-country. ${ }^{9,10}$

In 2002 the National Council of the Italian National Federation of Colleges of MDs and Dentists (FNOMCeO; www.fnomceo.it); selected and accredited nine types of CAM practices (homeopathy; acupuncture; Anthroposophic medicine; homotoxicology; chiropractic; osteopathy; TCM and ayurvedic medicine); and considered them all to be part of medical practice. In 2009 the same Council promulgated The Guidelines of the FNOMCeO on Non-Conventional Medicines and Practices. In addition two other documents in the same field were created; in 2011 by FISM (Federation of Italian scientific societies
- www.fism.it) and in 2012 by State-Region Conference (www. statoregioni.it). During the last years; numerous attempts to classify and incorporate the various practices into one single definition have failed (i.e. Non-Conventional Medicine; Alternative Medicine; Complementary Medicine; and Integrative Medicine). The various practitioners; the wide range of experiences which they bring along and their need to each emphasize the unique approach of their specific discipline; seemed to make it impossible to come to an agreement on this topic. However; recently the term "Complementary and Alternative Medicine" has been increasingly used to represent the approach as a whole. Whereas the new term "Integrative Medicine" refers to a practice that combines conventional treatments with CAM treatments that have been proven safe and effective.

In paediatric patients CAM is often used as in adults. ${ }^{1,11}$ About $30-50 \%$ of children with acute as well as with chronic diseases may use CAM in different European countries. ${ }^{12-16}$ In a recent survey of literature and expert perspectives in 20 European countries the mean CAM use in children was $52 \%$ (range: $5 \%-90 \%)^{2}$. This shows that CAM is often used to treat children in Europe and plays a crucial role in health care systems of many European countries. Its role in Italy's health system is unclear. Its use in children and the stakeholders in CAM have not yet been described in a comprehensive review. Therefore the aim of the present study is to examine the frequency of CAM use indications for use of CAM; different therapies used and factors influencing CAM use in children in Italy. Local experts in this field of medicine were asked to give a narrative overview of the use of CAM; its facilities and to describe the every-day CAM practice in paediatrics. 


\section{Methods}

A narrative; non-systematic review was assembled by combining expert opinions with a thorough and balanced review of available evidence from the literature (JGIM). An unrestricted literature search using the keywords; "alternative"; "complementary"; "integrative"; "prevalence" and "paediatric" or "children" and "Italian" was performed by internet search in March; 2011 using PubMed and Google Scholar. In addition; known local paediatric CAM experts were asked to provide additional articles and information in their native language about CAM use in their country. Their expertise about the healthcare policy and healthcare system in Italy was included. Furthermore; integration of CAM within different levels of healthcare and its legal aspects were described. Thereby information from peerreviewed and local journals could be considered as well as expertise from Italian paediatric CAM experts because some information about paediatric CAM-use in Italy was not published in English and/or not in indexed journals.

\section{Results}

\section{Characteristics of CAM use in adults and children in Italy}

In Italy; several surveys have been realized to investigate CAM use in children and adults ( Table 1). Twenty-two surveys were found between 1995 and 2012; published by international scientific journals or local journals in the Italian language. Twelve surveys included only adults; two presented investigations in all family members including children and eight surveys were specific for paediatric patients only. The most important and most representative survey was published in the year 2007 by the National Institute of Statistics. ${ }^{17}$ The study showed that in 2005 approximately 7.9 million persons; $14 \%$ of the entire Italian population; had used some of the following CAM during the three years preceding the investigation: homeopathy; phytotherapy; acupuncture and manual treatments. Other methods were not considered. An even higher CAM use could be shown in other surveys conducted on adults and including all CAM therapies: earliest surveys showed a prevalence of $30 \%$ in 1997 and $28 \%$ in $1999 .^{18,19}$ Later on; surveys in 2003 showed a CAM use of $31 \%{ }^{20}$ and $36 \%$; in $200447 \%{ }^{21}$ and in $200648 \% .^{22}$ Three surveys in 2009-11 showed a CAM use of $24 \% ; 28 \%$ and $34 \% .{ }^{23-25}$ Most frequent users were middle-aged women with higher educational as well as economic level. ${ }^{26}$ The survey has depicted some relevant differences about the use of CAM among Italian regions or areas: the highest percentage in the North East (11.4\%); followed by the North West (10.2\%); the Centre (6.8\%); the Isles (3.4\%) and the South $(2 \%) .{ }^{17}$ Most patients chose CAM based on the impression that a natural treatment is linked to higher safety with less negative side-effects; whereas allopathic and pharmacological treatment is linked to a fear of strong side effects and a general sense of discomfort. $48.8 \%$ of the respondents expressed a positive opinion about the usefulness of CAM treatment; while $51.2 \%$ deemed it as not useful. $60 \%$ of CAM users said that they had benefited from this type of therapy.

A similarly frequent use of CAM could be shown in children. The ISTAT survey also showed that $9.6 \%$ of children between $0-14$ years had used the three investigated CAM methods; homeopathy was represented with the highest percentage $(7.9 \%)$; followed by phytotherapy $(2.1 \%) .{ }^{17}$ In accordance with adults; only four CAM therapies were included in this survey. In addition to this; other studies have shown higher percentages. The largest survey specific for CAM use in paediatrics was conducted by Trapani et al. ${ }^{27}$ In the year 2000 the authors surveyed 3057 respondents in various Italian regions with paediatric patients from birth to 14 years of age. They reported that $46 \%$ of respondents use CAM for the health problems of their child $(56.1 \%$ male and $43.3 \%$ female). Furthermore this study shows a direct correlation between the use of CAM and the cultural level of the parents; which is in accordance to the ISTAT data. A recent survey conducted by Marchisio et al., ${ }^{28}$ in 2009 involved children (1-7years old) affected by recurrent acute otitis media ( $>3$ episodes in 6 months). It confirmed a high use of CAM (46\%) treatment with homeopathy and phytotherapy for chronic and recurrent diseases also confirmed by Trapani et al., ${ }^{29}$ especially for children at a young age.

A recent multicentre survey was conducted via a questionnaire filled out by parents of children admitted (as in-patients or as outpatients) to five general hospitals in Calabria (a region in the South of Italy).The Authors evaluated 1366 questionnaires which pointed out that from $18 \%$ (in Crotone) to $38 \%$ (in Cosenza) of the children used CAM. Phytotherapy (53-80\%) was preferred to homeopathy $(17-47 \%)$; this result stands in contrast to those registered in other regions. This is most likely given to the fact that in Calabria; CAM is prescribed more frequently by paediatricians; while self-medication is less common than in other regions. ${ }^{30}$ To estimate CAM use in chronic and severe diseases; a study involving children with blood cancer was of relevance: Clerici et al., ${ }^{31}$ determined that twelve percent of the children had used at least one kind of CAM during conventional treatment. $83 \%$ reported benefits such as prevention of chemotherapy side effects.

CAM use in different age groups has been investigated in several studies. The survey performed by ISTAT showed that CAM is used most frequently in the age groups from 3 to 5years (11\%); while for the other age groups it was $6 \%{ }^{17}$ Similar results were found in a survey by Trapani et al., ${ }^{32}$ CAM use in children 0 -2years was $31 \%$; in 3 -5years $31 \%$; in $6-10$ years $26 \%$ and in $11-14$ years $11 \%{ }^{27}$

CAM is a heterogeneous group of various therapies and remedies. In Italy homeopathy has a long tradition and seems to be very popular. Its use in adults may vary between $27 \%{ }^{19}$ and $30 \% .^{33}$ Interestingly; results of reviewed surveys showed that phytotherapy has an even higher prevalence in adult CAM users and may vary between $47 \%$ and $52 \%$.

In children the use of homeopathy is very high (up to $72 \%$ of all CAM users). ${ }^{27}$ Herbal medicine is the second most frequently used CAM therapy in children (up to a mean value of $66 \%{ }^{30}$ This ranking is confirmed by data of Trapani et al.; where the most commonly used CAM in children was homeopathic medicine $72 \%$; followed with a large distance by phytotherapy (17\%); acupuncture (5\%); Anthroposophic medicine (2\%); chiropractic (2\%) and osteopathy. ${ }^{27}$ This stands in partial contrast to the results of two recent surveys; conducted on patients ${ }^{30}$ and on paediatricians; ${ }^{32}$ which both demonstrated the predominance of phytotherapy. The herbal remedies mentioned were Echinacea purpura; chamomile; liquorice; fennel; aloe; valerian; almond oil; propolis and cranberry. Manual Therapies were the third most frequent CAM. Acupuncture and other oriental medicine were not really used in children (use of all CAM therapies $1-6 \%) \cdot{ }^{17,27}$

Certain studies provide data about diseases treated by CAM. Mentioned were ENT (eye; nose; and throat) diseases; allergies; skin disorders; sleep disorders; lung diseases and gastrointestinal diseases as well as alimentary disorders (see Table 1 for the specific percentage). ${ }^{30}$ Highest CAM use was found in ENT diseases (about $30 \%$ ) explained by the fact that this type of disease is very common among children attending community childhood care. ${ }^{29,30}$ 


\section{CAM and how it is handled by general practitioners and paediatricians}

General practitioners (GPs) play a central role in health care by usually acting as the first medical professional to discuss the patient's health as well as the use of CAM. Therefore the handling of CAM by paediatricians and GPs is of high interest. Some characteristics of physician's handling with CAM are known through a survey conducted on Italian general practitioners. ${ }^{34}$ Most of the responding GPs (58\%) recommended CAM; but only a far smaller fraction (13\%) practised it; $36 \%$ of CAM practitioners had no certified training. Being female; younger age; practising in larger communities; having had some training in CAM as well as following a vegetarian or macrobiotic diet and engaging in physical activity were independent predictors of CAM recommendation and practice. $42 \%$ of GPs did not recommend CAM to patients at all; mostly due to insufficient evidence for its effectiveness.

Clearly the trend towards use of CAM in physicians is growing. A recent publication ${ }^{35}$ shows that in Italy more than 20;000 practitioners use; apply and/or prescribe homeopathic remedies; acupuncture; Anthroposophic medicine and phytotherapy. A good percentage of them have completed years of post-graduate training. It is assumed that the number of MDs who use CAM on occasion is increasing due to the fact that many preparations of plants; probiotics and essential fatty acids; are classified as dietary supplements and are thus more commonly used. A recent survey (online questionnaire) of paediatricians enrolled in the Italian Society of Paediatrics (SIP) shows that about $25 \%$ routinely uses complementary therapies and about $90 \%$ prescribes both CAM and conventional therapies. Phytotherapy (84\%) and homeopathy (77\%) were the most commonly used complementary therapies. Among the paediatricians who do not prescribe non-conventional therapies $1 \%$ consider CAM as harmful; $65 \%$ as ineffective and $33 \%$ are just not interested in these therapies. ${ }^{32}$

\section{CAM treatment offers in outpatient clinics and inpatient wards}

Outpatient clinics and inpatients wards offering CAM are rare in most European countries and most CAM therapies are applied by family doctors and paediatricians in primary care. ${ }^{2}$ In Italy; such physicians are represented numerously and allocated in all regions. However; some outpatient clinics and wards with CAM therapies were assessable as well. In all of Italy 45 known bigger clinics and hospitals offer CAM. Thirty-eight offer acupuncture; six offer homeopathy; three offer phytotherapy and some others offer different forms of CAM ( Table 2 ). All institutions were conceived for adults. There are no children's hospitals in Italy dedicated to CAM. But there are many smaller outpatient services offering CAM in numerous Italian regions that additionally treat paediatric patients. These structures are fairly well distributed throughout Italy and can be found increased in Piedmont; Emilia Romagna; Tuscany; Lazio and Campania.

Although no official CAM treatment exists in Italian paediatric hospitals; several projects involving CAM were carried out or are ongoing. A number of studies on Phytotherapy (RCT and retrospective) were conducted involving children affected by recurrent urinary infections (RVU) and/or vesicoureteral reflux (VUR) that were treated with cranberry. ${ }^{36}$ Currently a randomized controlled blinded study is being conducted involving more than 200 children affected by recurrent urinary tract infections to test treatment with standardized extract of cranberry versus treatment with antibiotics. In the clinic of neonatology of Turin; Savino et al. conducted a survey involving infants affected by abdominal colic; using an extract made with lemon balm; fennel and chamomile. ${ }^{37}$ Recently; a survey was conducted in the Children's Hospital of Turin; to study the effect of Monascus purpureus (red yeast rice) on hypercholesterolemic children. ${ }^{38} \mathrm{~A}$ study regarding homeopathy; was recently conducted on the modulation of cutaneous allergic inflammation in children using the remedy Apis. ${ }^{32}$ As a result of a controlled clinical study; the use of a preparation of Arnica and Echinacea for the treatment of the new-born umbilical stump is still very common in the Clinic for Neonatology of Novara. ${ }^{39}$ Furthermore; CAM is utilized for psychological disorders such as attention- and behavioural deficits in the same hospitals (not published data). These examples show that the prescription of CAM in children is still a prerogative for medical private practices; although an initial interest and implementation is visible in public hospitals.

\section{Education programs and work-groups in CAM}

Twenty-five education programs in CAM were found. Six of these were master courses; one in homeopathy; and two in acupuncture respective TCM and three in phytotherapy ( Table 3 ). They were allocated in all regions and most of them were of great popularity. None of them were dedicated to paediatric medicine; but most of them included training in treatment of children. Furthermore; many national societies on CAM such as the Italian Society of Homeopathy and Integrated Medicine (SIOMI); the Italian Society of Natural Medicine (SIMN) and others; are involved in studies and trials on childhood care. Paediatric societies like the Italian Society of Paediatrics (SIP) and the Italian Federation of Paediatricians (FIMP) have work-groups focused on CAM. In particular; the SIP has set up a commission on CAM with the goal to quantify the use of CAM in paediatrics in the country; to promote clinical trials testing its efficacy; assessing the adequacy of CAM in the field of health protection of the child and to provide data on National Health Service's costs of these practices.

Previously; FIMP has organized a network of excellence on herbal medicine; with the aim of spreading knowledge about a scientific and rational use of herbal medicine in children; according to the EvidenceBased-Medicine (EBM) criteria; with particular attention to possible interactions and side-effects of medicinal plants. This network has organized numerous training courses for paediatricians and family doctors and in 2011 it released the first edition of the "School of Herbal Medicines and Nutritional Supplements".

\section{Political aspects of CAM in Italy}

Currently there is no legislation regulating practices of CAM; despite of numerous bills that have been proposed in the recent decade. The first bills were advanced in 1984 with the intent to regulate Asian medicine. In 1985 a proposal was made to establish the occupational image of energy flow therapists; which was amended in 1988 and 1994. Even the most recent bills incurred by a number of efforts and intentions for the regulation of CAM have failed to find approval (Galletti in 1997; bill n.3891; Lucchese in 2006; bill $\mathrm{n} .874$ and Bosone in 2008; bill n. 714). The last bills were proposed in 2011 (Bill n. 2672 and Bill n. 2673) with the intent to obtain a regulation for TCM and acupuncture in the first case and homeopathy in the second case; however; both are still pending. The only laws that were enacted in the years to cover the regulatory gaps were laws enacted on a regional level. The region at the forefront for CAM is Tuscany; where the establishment of tools for integration of "Non-ConventionalMedicine" (NCM) into health interventions was initially approved of by regional law in 1998. CAM practices are included in the "Essential Level of Assistance" (LEA) in Tuscany now and settled by the "National Sanitary System" for acupuncture; homeopathy; herbal 
medicine and TCM. In 2007 the Regional Council of Tuscany passed a law (Regional Law n.9/2007) that regulates the practice of CAM on the part of MDs; dentists; VDs and pharmacists. The following year (2008) a Protocol of Agreement was signed by Tuscany Region and Professional Associations. Practices of CAM are regulated by the "Code of Ethics" as "art. 15 states": "The use of non-conventional practices cannot be separated from respect for the decorum and dignity of the profession and is expressed in the exclusive area of the physician's professional responsibility; which is direct and nondelegable. The use of unconventional practices; however; should not reduce the citizen to specific treatments; it should be scientifically established and requires detailed information and acquisition of consent. The doctor is prohibited from working towards or facilitating the exercise of third non-medical practices in the field of so-called unconventional treatments." In sum; Italy has no national legislation on CAM; even though there are some theoretical limitations; physicians can treat patients with CAM practically without limitations.

\section{Discussion}

CAM is widely used in Europe and the awareness for CAM in healthcare and politics is increasing. ${ }^{2}$ In Italy CAM use seems to be high as well. Based on published surveys analysed in our review it can be estimated that $30-48 \%$ of Italian citizens use CAM and that CAM-use by children is of similar extent. These results are vague because patients self-administer and CAM is not financed by health insurances and thus relevant data cannot be verified by other sources such as data from health-insurances etc. Some surveys show that a large percentage of CAM users do not disclose their CAM-use to physicians which increases risk for interactions with pharmaceutical treatments and missed side-effects. ${ }^{31}$

Especially in obstetrics; different researchers emphasize that the use of herbal products during pregnancy; as is common among Italian women; is not always appropriate and in some cases potentially harmful. ${ }^{22}$

Phytotherapy; homeopathy and acupuncture are the most preferred CAM therapies of the Italian population. Phytotherapy has a long tradition in all regions of Italy; and is called "folk medicine". A recent interest of health care providers and of industries in herbal products has dramatically increased and consequently a lot of research is developing with the goal to scientifically verify the positive effects indicated by traditional knowledge. Even if there are only few products registered as herbal drugs; hundreds of dietary supplements contain phyto-pharmaceutical components and all these products are available prescription-free in pharmacies; herbal stores and also in alimentary stores. Review articles indicate that phytotherapy is the most frequently used CAM therapy in Italy. ${ }^{24,40}$ Nevertheless; our review shows that homeopathy is the most implemented CAM in children. Although articles appear regularly in the press refuting evidence and efficacy of homeopathy by emphasizing a lack of controlled clinical trials; its popularity and its use remain high. Perhaps its low harmpotential encourages parents to self-administer homeopathic remedies in children. The use of TCM and oriental medicine; such as Ayurveda; may have increased in the last decades as well; but its prevalence in reviewed surveys is relatively small.

Many Italian regions have introduced CAM services in public clinics as a response to publications of Italian surveys showing a high CAM use. Interestingly; most services offer acupuncture; although its prevalence in Italy is rather small in contrast to phytotherapy and homeopathy. This may be explained by the fact; that homeopathy and phytotherapy are often used without prescription and without a therapist; whereas acupuncture is usually practiced in designated institutions only. Additionally; this apparent increase of TCM is unrealistic; due to the fact that most education programs and CAM master degrees include homeopathy and phytotherapy and not acupuncture. This leads to the assumption that most CAM therapists use homeopathy and phytotherapy. ${ }^{41}$

Italy still has no national legislation on CAM. The advent of federalism in health matters has meant that there are huge differences between regions. The most striking example is Tuscany; where CAM is practiced in more than 100 affiliated clinics and where a hospital is to be opened in the near future. In this region; the visits are funded totally or partially by the National Health Service. The use of therapies (conventional or CAM) are influenced by the public perception. This clearly weakens the concept of health as a right guaranteed to every human being under the care of freedom enshrined in the constitution. The health of the population; its humanization; personalization and the sustainability of treatment all have the patient's choice of treatment at their core. Discussions about legality of CAM in Italy are creating conflict. There are no medical-legal restrictions in the practice of CAM; except those that automatically go along with being a registered physician. In fact; the main regulatory reference is Article 348; the "penal code" (abuse of the medical profession) that regulates its use. The only limit to this type of practice is that it lies in the direct and non-delegable responsibility of the physician to evaluate; re-evaluate and improve decision-making from case to case and to compensate and supplement the available scientific knowledge. CAM practitioners must keep in mind what can be considered "scientifically valid" and they are responsible for presenting and implementing "alternative options". Of course; prerequisite for the legitimacy of any treatment is still the patient's consent. Especially when CAM treatment is an option the patient must be properly informed on the available alternatives (conventional and unconventional) as well as the expected results and possible consequences arising from the exclusion of conventional therapy. The risks and the costs of the chosen treatment; the knowledge on which it is based; how it is structured and the possible lack of experimental verification need to be made clear to the patient.

In sum; CAM use in Italy seems to be as high as in other European countries; both in adults and children. However; in contrast to most other countries; several surveys have been conducted to describe CAM use and multiple efforts have been made to clarify its role in the health system. Local experts hope that in accordance with European Union directives; Italy will receive a national law; based on evidence and supported by scientific research that will allow physicians to finally speak of medicine as a whole; without having to explicitly distinguish between conventional and alternative medicine. A national review about CAM use in children in Italy currently does not exist. Because of this lack of comparable surveys; it was not possible to perform a systematic review. By melding published surveys with the opinions of local paediatric CAM experts; a survey on the use of CAM by children in Italy could be conducted for the first time. The quality of the data is limited because of lack of a general definition of CAM use and inquired CAM therapies. Therefore; a definition of CAM use has to be generated and a list of CAM therapies has to be provided; which both have to be included in future questionnaires. We hope that our review of the status quo encourages experts and politicians to initiate next steps towards scientific based and unified handling of CAM.

\section{Acknowledgments}

None. 


\section{Conflicts of interest}

Author declares there are no conflicts of interest.

\section{Funding}

None.

\section{References}

1. Molassiotis A, Fernadez-Ortega P, Pud D, et al. Use of complementary and alternative medicine in cancer patients: a European survey. Ann Oncol. 2005;16(4):655-663.

2. Zuzak TJ, Boňková J, Careddu D, et al. Use of complementary and alternative medicine by children in Europe: Published data and expert perspectives. Complement Ther Med . 2013;1:S34-47.

3. Längler A, Zuzak TJ. Complementary and alternative medicine in paediatrics in daily practice-A European perspective. Complement Ther Med. 2013;1:S26-33.

4. Kemper KJ, Vincent EC, Scardapane JN. Teaching an integrated approach to complementary; alternative; and mainstream therapies for children: a curriculum evaluation. J Altern Complement Med. 1999;5(3):261-268.

5. Kemper KJ, O'Connor KG. Pediatricians' recommendations for complementary and alternative medical (CAM) therapies. Ambul Pediatr. 2004; 4(6): 482-487.

6. Jobst D, Niebling W. [Naturopathic methods as part of the academic curriculum and the role of general practice]. Forsch Komplementarmed Klass Naturheilkd. 2005;12(5):272-276.

7. Brinkhaus B1, Witt CM, Jena S, Bockelbrink A, et al. Integration of complementary and alternative medicine into medical schools in Austria; Germany and Switzerland - Results of a cross-sectional study. Wien Med Wochenschr. 2010;161(1-2):32-43.

8. CAMbrella. European research network for complementary and alternative medicine. 2011.

9. World Health Organization (WHO). WHO Agenda item 12.4 Traditional medicine. World Health Organization technical report series, Geneva. 2009 .

10. Pritchard-Jones K, Lewison G, Camporesi S, et al. The state of research into children with cancer across Europe: new policies for a new decade. Ecancermedicalscience. 2011;5:210.

11. Kemper KJ, Vohra S, Walls R. American Academy of Pediatrics. The use of complementary and alternative medicine in pediatrics. Pediatrics. 2008;122(6):1374-1386.

12. Vlieger AM, van de Putte EM, Hoeksma H . The use of complementary and alternative medicine in children at a general paediatric clinic and parental reasons for use. Ned Tijdschr Geneeskd; March. 2006;150(11):625-630.

13. Laengler A1, Spix C, Seifert G, et al. Complementary and alternative treatment methods in children with cancer: A population-based retrospective survey on the prevalence of use in Germany. Eur J Cancer. 2008;44(15):2233-2240.

14. Zuzak TJ1, Zuzak-Siegrist I, Simões-Wüst AP, Rist L, et al. Use of complementary and alternative medicine by patients presenting to a Paediatric Emergency Department. Eur J Pediatr.2009;168(4):431-437.

15. Vlieger AM, van Vliet M, Jong MC. Attitudes toward complementary and alternative medicine: a national survey among paediatricians in the Netherlands. Eur J Pediatr. 2010;170(5):619-624.

16. Gottschling S, Gronwald B, Schmitt S, et al. Use of complementary and alternative medicine in healthy children and children with chronic medical conditions in Germany. Complementary Therapies in Medicine accepted Complement Ther Med. 2011;1:S61-69.
17. ISTAT. Le terapie non convenzionali in Italia (Indagine multiscopo sulle condizioni di salute e ricorso ai servizi sanitari 2005. ISTAT Istituto Nazionale di statistica Roma. 2007.

18. Dello Buono M, Urciuoli O, Marietta P, et al. Alternative medicine in a sample of 655 community-dwelling elderly. J Psychosom Res .2001;50(3):147-154.

19. Menniti-Ippolito F, Bologna E, Gargiulo L, et al. Characteristics of unconventional medicine users and their households in Italy. Ann Ist Super Sanita. 2004;4(40):455-461.

20. Rossi P, Di Lorenzo G, Malpezzi MG, et al. Prevalence, pattern and predictors of use of complementary and alternative medicine (CAM) in migraine patients attending a headache clinic in Italy. Cephalalgia. 2005;25(7):493-506.

21. Zaffani S, Cuzzolin L, Benoni G. Herbal products: behaviors and beliefs among Italian women. Pharmacoepidemiol Drug Saf. 2006;15(5):354-359.

22. Lapi F, Vannacci A, Moschini M, et al. Use, Attitudes and Knowledge of Complementary and Alternative Drugs (CADs) Among Pregnant Women: a Preliminary Survey in Tuscany. Evid Based Complement Alternat Med. 2006;7(4):477-486.

23. Bertomoro P, Renna S, Cottone $\mathrm{M}$, et al. Regional variations in the use of complementary and alternative medicines (CAM) for inflammatory bowel disease patients in Italy: an IG-IBD study. J Crohns Colitis. 2009;4(3):291-300.

24. Cardini F, Lesi G, Lombardo F, et al. The use of Complementary and Alternative Medicine by women experiencing menopausal symptoms in Bologna. BMC Womens Health. 2010;10:7.

25. Cuzzolin L, Francini-Pesenti F, Verlato G, et al. Use of herbal products among 392 Italian pregnant women: focus on pregnancy outcome." Pharmacoepidemiol Drug Saf. 2010;19(11):1151-1158.

26. Barbadoro P, Chiatti C, Errico MD, et al. Complementary and Alternative Medicine (CAM) among adults in Italy: Use and related satisfaction." European Journal of Integrative Medicine. 2011;3(4):e325-e332.

27. Trapani G, Barbato C, Zanino L, et al. Uso delle Medicine Non Convenzionali nella poplazione pediatrica dell'Italia Nord-Occidentale. La proffesione. 2002.

28. Marchisio P, Esposito S, Bianchini S, et al. Effectiveness of a propolis and zinc solution in preventing acute otitis media in children with a history of recurrent acute otitis media. Int J Immunopathol Pharmacol. 2010;23(2):567-575.

29. Trapani G, Alfonso DG, Antonio L, et al. Indagine nella Regione Liguria sull'uso delle Medicine Non Convenzionali in Pediatria. Cahiers de Bioterapie ed. Italiana. 2000;4:39-45.

30. Dolceamore TR, Altomare F, Zurlo F, et al. Use of alternativecomplementary-medicine (CAM) in Calabrian children. Ital J Pediatr. 2012;38:70.

31. Clerici CA, Veneroni L, Giacon B, et al.Complementary and alternative medical therapies used by children with cancer treated at an Italian pediatric oncology unit. Pediatr Blood Cancer. 2009;53(4):599-604.

32. Macrì F AL, Careddu D, Cocchi G, et al. Attitude of Italian pediatricians towards Complementary Medicines. ECIM. 2012.

33. Johannessen H, von Bornemann Hjelmborg J, Pasquarelli E, et al. Prevalence in the use of complementary medicine among cancer patients in Tuscany; Italy. Tumori. 2018;94(3):406-410.

34. Giannelli M, Cuttini $M$, Da Frè $M$, et al. General practitioners' knowledge and practice of complementary/alternative medicine and its relationship with life-styles: a population-based survey in Italy. BMC Fam Pract. 2007;8:30 
35. Sarsina PR, Iseppato I. Non-conventional medicine in Italy: the present situation. European Journal of Integrative Medicine. 2009;1:65-71.

36. Ferrara P, Romaniello L, Vitelli O, et al. Cranberry juice for the prevention of recurrent urinary tract infections: a randomized controlled trial in children. Scand J Urol Nephrol. 2009;43(5):369-372.

37. Savino F, Cresi F, Castagno E, et al. A randomized double-blind placebo-controlled trial of a standardized extract of Matricariae recutita; Foeniculum vulgare and Melissa officinalis (ColiMil) in the treatment of breastfed colicky infants. Phytother Res. 2005;19(4):335-340.

38. Guardamagna $\mathrm{O}$, Abello $\mathrm{F}$, Baracco $\mathrm{V}$, et al. The treatment of hypercholesterolemic children. Efficacy and safety of a combination of red yeast rice extract and policosanols. Nutr Metab Cardiovasc Dis. 2011;21(6):424-429.
39. Guala A, Pastore G, Garipoli V, et al. The time of umbilical cord separation in healthy full-term newborns: a controlled clinical trial of different cord care practices. Eur J Pediatr. 2003;162(5):350-351.

40. Cuzzolin L, Francini-Pesenti F, et al. Use of herbal products among 392 Italian pregnant women: focus on pregnancy outcome. Pharmacoepidemiol and Drug Saf. 2010;19(11):1151-1158.

41. http://www.jgimed.org/ 